\title{
Study on the Retention Behavior of Aromatic Carboxylic and Sulfonic acid on a New Anion Exchange Column
}

\author{
SHI, Ya-Li(史亚利) CAI, Ya-Qi((蔡亚岐) MOU, Shi-Fen(牟世芬) \\ State Key Laboratory of Environmental Chemistry and Ecotoxicity, Research Center for Eco-environmental \\ Sciences, Chinese Academy of Sciences, Beijing 100085, China
}

\begin{abstract}
Ion chromatography (IC) has gradually developed into a preferred method for the determination of inorganic anions. And in recent years some low molecular aliphatic acid can be also separated in the ion exchange column with the development of stationary phase. But for the determination of aromatic ionic compounds there are some problems. The aromatic anions show enhanced retention due to interaction with the $\pi$ electrons of the aromatic backbone. Although the addition of an organic modifier can alleviate the difficulty, it is not the ultimate solution. IonPac AS20 column was developed using a unique polymer bonding technology and its substrate coating is aliphatic backbone. The polymer is completely free of any $\pi$ electron-containing substituents in the AS20 column. In this paper, the retention behavior of aromatic carboxylic and sulfonic acid on two hydroxide-selective columns, IonPac AS11-HC, AS16, and the new column AS20 was also studied. The result showed that the retentions of ten compounds on three columns were different with each other because of their different column characteristics. Among them 4-chlorobenzene sulfonic acid, 3,5-dihydric benzoic acid and salicylic acid obviously exhibited the weakest retention on the IonPac AS20. It was showed that $\pi-\pi$ bond function between anion and stationary phases was weakened in AS20 column because its polymer was completely free of any $\pi$ electron-containing substituents. So in this paper the AS20 was selected as an analytical column to separate ten aromatic ionic compounds, fumaric acid with conjugate bond included. The retention behavior, separation of the ten compounds and effect of temperature on their retention in the anion-exchange column AS20 $(2 \mathrm{~mm})$ were studied. The result showed that those compounds could be separated with each other when running in gradient program and the organic modifier was unnecessary during the separation. So it is showed that AS20 column can be used as a separating column because its polymer is completely free of any $\pi$ electron-containing substituents. Finally, the effect of temperature on the retention behavior in AS20 column was studied and it was showed that the retention of nine compounds exhibited endothermic behavior.
\end{abstract}

Keywords aromatic carboxylic acid, aromatic sulfonic acid, ion chromatography

\section{Introduction}

Aromatic compounds are important chemical substances and additives in many industrial processes and products. In the pharmaceutical industry, benzoic acid and its derivatives are used as antiphlogistic, antiseptic, analgesic, antipyretic, antirheumatic and anaesthetic drugs. ${ }^{1}$ Sodium benzoate is often used as a preservative additive in the food industry because it can inhibit microorganism. Benzene sulfonic acids (BS) are used in the manufacture of azo dyestuffs, pharmaceuticals and tanning agents. ${ }^{2}$ Additionally, aromatic carboxylic acids are also widely used in the production of thermostable polymers and they are important semi-products of biosynthesis of aromatic amino-acids in plants (phenolic acids), metabolites of numerous toxic substances, drugs and catecholamine. ${ }^{1,3}$ Many manufactures discharge their wastewater into rivers after a treatment process to eliminate the most common contaminants. But the benzene sulfonic and carboxylic acids are difficultly biodegraded in wastewater treatment plants and they are very soluble in water. ${ }^{4}$ So they cannot be removed by a common treatment method and are easy to enter the river. They gradually enter the groundwater, soil and so on. So the analysis of aromatic acids, including aromatic carboxylic acid and sulfonic acid, is receiving considerable attention gradually.

There are many methods developed for the determination of aromatic carboxylic and sulfonic acid, such as liquid chromatography (LC), ion-pair liquid chromatography (IPC) with UV-Vis detection, capillary electrophoresis (CE), gas chromatography (GC) and the methods based on the MS as a detector. ${ }^{5-10}$ For the anion analysis, ion chromatography (IC) has its particular predominance and has gradually developed into the preferred method for the determination of anions since it

* E-mail: caiyaqi@rcees.ac.cn

Received April 30, 2007; revised July 10, 2007; accepted September 19, 2007.

Project supported by the National Basic Research Program of China (No. 2003CB415001), the National Natural Science Foundation of China (Nos 20475060, 20621703) and Major Research Program of Chinese Academy of Sciences (No. KZCX3-SW-432). 
was introduced in 1975. For the determination of weak organic acid, ion exclusion chromatography rather than ion-exchange chromatography was often selected as an analytical method. But for the determination of aromatic acid there are only few reports ${ }^{11-13}$ and the organic modifier is necessary. IEC will be unsatisfied for simultaneous analysis of fully ionized strong acids, such as aromatic sulfonic acid and inorganic anions. Additionally, ion-pair chromatography (IPC) can be also used to separate aromatic ion compounds. But it is difficult to select suitable ion-pair reagent for some compounds. And the eluent used in IPC was more complex and expensive than in ion-exchange chromatography. So compared with IEC and IPC, ion-exchange chromatography will have its predominance in the application of aromatic ion compound analysis. With the development of stationary phase, ion-exchange chromatography has also been used in the simultaneous determination of some low molecular aliphatic acid and inorganic anions. ${ }^{14-16}$ Sarzanini and co-workers ${ }^{17}$ studied the separation and retention behavior of carboxylic acids in IonPac AS11. And it was also reported that inorganic anion, aliphatic acid and aromatic acid had been separated in anion-exchange column, ${ }^{18}$ but the eluent with higher concentration was necessary and the peak of aromatic acid was tailed.

Why was IC not widely used in the separation of aromatic ionic compounds? And what is the difficulty in that analysis? The possible reason is that the separation mechanism is not only ion exchange but also the stronger hydrophobic interaction of the analytes with the resin. It was reported that the retention of polarizable anions was strong and the peaks was tailed due to the presence of polydivinylbenzene polymeric matrix. ${ }^{19}$ Fritz $^{20}$ also suggested the strong retention of polarizable anions should be the result of the combination effects of hydrophobic attraction, hydrogen bonding and lower dielectric constant. In fact the matrix of anion exchangers is almost polydivinylbenzene polymer and most of aromatic ionic compounds are polarizable. So the aromatic anions show enhanced retention due to interaction with the $\pi$ electrons of the aromatic backbone. Consequently, the presence of above problem is inevitable when aromatic ionic compounds are separated in anion-exchange column. Although the addition of an organic modifier can alleviate the difficulty, it is not the ultimate solution.

IonPac AS20 column was developed using a unique polymer bonding technology and its substrate coating is aliphatic backbone. The polymer is completely free of any $\pi$ electron-containing substituents in the AS20 column. ${ }^{21}$ Therefore the $\pi-\pi$ bond action between the aromatic anions and stationary phase will not exist. In this paper the retention behavior on three hydrophilic columns was studied. And then IonPac AS20 column was selected for the separation of ten aromatic ion compounds. The effect of temperature on the retention in the IonPac AS20 column was also studied. The gradient eluent program was optimized and the ten compounds could be separated baselinely when the eluent was run in this gradient program.

\section{Experimental}

\section{Reagents}

All reagents were of analytical reagent-grade unless specified otherwise. All solutions were prepared with deionized water further purified by an EASYpure LF system (Barnstead, USA) with a specific resistance of $18.3 \mathrm{M} \Omega \bullet \mathrm{cm}^{-1}$. 4-Chlorobenzene sulfonic acid (4-CBSA, 3), 3,5-dihydroxybenzoic acid (3,5-DHBA, 10) and pyridine-2,6-dicarboxylic acid (Py-2,6-DCA, 7) were purchased from Aldrich corporation (USA); $p$-aminobenzene sulfonic acid ( $p$-ABSA, 2), fumaric acid (FA, 5), and salicylic acid (SCA, 6) were obtained from Beijing Chemical Plant (Beijing, China); 4-hydroxybenzoic acid (4-HBA, 8), benzoic acid (BA, 1), $p$-phthalic acid $(p$-PA, 9), $o$-phthalic acid (o-PA, 4) and 50\% sodium hydroxide solution were purchased from Fluka corporation (USA).

All standard stock solutions at $1000 \mathrm{mg} / \mathrm{L}$ were prepared with deionized water. For those higher hydrophobic compounds methanol was added into the solution in order to make them dissolve. Or $\mathrm{pH}$ of the solution was adjusted to make some compounds dissolve. Standard solutions were prepared daily by serial dilution of the stock solution prior to use.

\section{Instruments and chromatographic condition}

A Dionex model ICS2500 ion chromatography (Sunnyvale, CA, USA) equipped with a GS50 pump, an LC25 column oven, an ED50A detector was used. IonPac AS20 $(2 \mathrm{~mm} \times 250 \mathrm{~mm})$, AG20 $(2 \mathrm{~mm} \times 50 \mathrm{~mm})$, IonPac AS11-HC ( $2 \mathrm{~mm} \times 250 \mathrm{~mm})$, AG11-HC $(2 \mathrm{~mm}$ $\times 50 \mathrm{~mm})$, IonPac AS16 $(2 \mathrm{~mm} \times 250 \mathrm{~mm})$ and AG16 columns $(2 \mathrm{~mm} \times 50 \mathrm{~mm})$ were used throughout. The eluent was sodium hydroxide at different concentrations, which was run at a flow-rate of $0.25 \mathrm{~mL} / \mathrm{min}$. The solution of $\mathrm{NaOH}$ was prepared by dilution of a $50 \% \mathrm{NaOH}$ stock solution. The detection was performed by a Dionex ED50A conductivity detector. Suppression of the eluent was achieved by a Dionex ASRS-ULTRA suppressor $(2 \mathrm{~mm})$ in the auto suppression external water mode. All tubing in the chromatography path (from the outlet of the pump to the exit of the suppressor) was of PEEK. The sample was loaded into the loop by an Autosampler AS50. Both instrument control and data collection were performed with a personal computer equipped with a Dionex Chromeleon chromatography workstation.

\section{Results and discussion}

\section{Comparison of retention behavior on three an- ion-exchange columns}

The IonPac AS20 column was a new anion-exchange column developed with a unique polymer bonding 
technology. The polymer of AS20 has excellent selectivity for hydroxide eluent because of its extreme hydrophilicity. And the polymer used in the AS20 column is completely free of any $\pi$ electron-containing substituents because its substrate coating is aliphatic backbone. Therefore the $\pi-\pi$ bond action between the aromatic anions and stationary phase will not exist.

In this paper two hydrophilic columns, IonPac AS16 and IonPac AS11-HC, were selected to compare with the new column AS20. The retention behavior of aromatic ionic compounds, including fumaric acid with conjugate bond, in the three columns was studied and discussed. Why did we select these two columns to compare with AS20? The reason is that AS11-HC column is often used to separate organic acids and AS16 is a highly hydrophilic column. So we thought that these two columns should be more appropriate for the separation of hydrophobic compounds than other anion exchange columns. Some characteristics of the three anion-exchange columns are listed in Table 1. Firstly, we studied the effect of $\mathrm{NaOH}$ on their retention in the three columns. The comparison chart for retention with $45 \mathrm{mmol} / \mathrm{L} \mathrm{NaOH}$ as an eluent is showed in Figure 1. Secondly the effects of acetonitrile on the retention in the three columns were studied because acetonitrile could prevent the action between the $\pi$ electron clouds of a compound and the aromatic backbone of the stationary phase. And the retention of hydrophobic compounds will be affected greater. The rate of their retention time decrease can reflect hydrophobic action between the compounds and the stationary phase. The comparison chart with the mixture of $30 \mathrm{mmol} / \mathrm{L} \mathrm{NaOH}$ and different concentration acetonitrile as an eluent is showed in Figure 2. As shown in Figure 2, the retentions of the selected compounds are all decreased with the content of acetonitrile increasing, which indicates that there is hydrophobic action between all compounds and the stationary phase. According to the rate of their retention time decrease the ten compounds can be divided into three groups. The first one is AS11-HC $>$ AS16> AS20 for $p$-ABSA, 4-CBSA, BA, $o$-PA, 3,5-DHBA and SCA. The second one is AS20 $>$ AS11-HC $>$ AS16 for fumaric acid and $p$-PA. And the last one is AS11-HC> AS20 $>$ AS16 for Py-2,6-DCA and 4-HBA. The $\pi-\pi$ bond action between the compounds in the first group and stationary phase is the strongest. And because of the disappearance of $\pi-\pi$ bond action, the hydrophobic action is weakened greatly as shown in Figure 2.

As indicated in the introduction, the chromatographic mechanism for aromatic ionic compounds mainly includes ion exchange and stronger hydrophobic

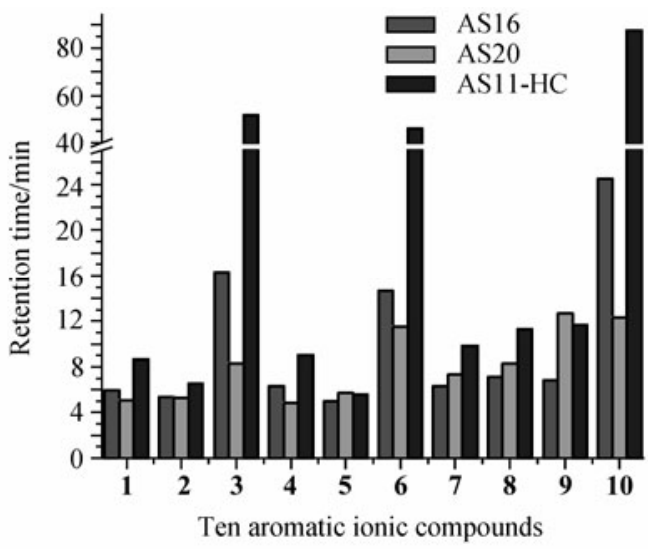

Figure 1 The retention behavior on the three different columns with $45 \mathrm{mmol} / \mathrm{L} \mathrm{NaOH}$ as an eluent at $30{ }^{\circ} \mathrm{C}$. All ionic concentrations are $40 \mathrm{mg} / \mathrm{L}$ except for that of 4-CBSA, which is 12.5 $\mathrm{mg} / \mathrm{L}$.

interaction of the analytes with the resin. So the reason for the above mentioned phenomenon existing may be that the retention mechanism for the ten ionic compounds is different from each other in the three columns. If the ion-exchange mechanism plays the major role, the retention order will be mainly dependent on the column capacity. But if the hydrophobic action is the major separation mechanism, the column hydrophobicity will play the major actions for the retention of analytes. The result of comparison study showed that hydrophobic action for the ten acids was different with each other. And they could be divided into three groups according to the comparison charts. For FA and $p$-PA, hydrophobic action may be the weakest because the rate of their retention time decrease was similar in the three columns. So their retention time was mainly dependent on the column capacity. The order of their retention in the three columns is AS20 $>$ AS11-HC $>$ AS16, the same as the column capacity. In contrast, the hydrophobic action for pyridine-2,6-dicarboxylic acid and 4-hydroxybenzoic acid was stronger. So their retention may be dependent on both the column capacity and hydrophobicity. And their retention was stronger in AS11-HC than in AS20 though the capacity of AS20 was larger than AS11-HC. For the other six compounds, the hydrophobic interaction between the analytes and the resin was the strongest and it played the important role for their retention. So the hydrophobicity of column affects their retention more greatly than the others. The order of column hydrophobicity is: AS11-HC $>$ AS16, AS20. There are no data indicating the order of hydrophobicity of AS16 and AS20 columns. But as indicated above, the substrate

Table 1 Comparison of the characteristics of three anion-exchange columns

\begin{tabular}{ccccccc}
\hline \multirow{2}{*}{ Column type } & \multicolumn{2}{c}{ Particle } & Column capacity & \multirow{2}{*}{ Hydrophobicity } & \multicolumn{2}{c}{ Latex } \\
\cline { 2 - 3 } & Diameter/ $\mu \mathrm{m}$ & Porosity & Dequiv./column) & & Diameter/nm & Cross-linking \\
\hline AS20 & 7.5 & Super-macroporous & 77.5 & Ultralow & grafted & grafted \\
AS11-HC & 9.0 & Super-macroporous & 72.5 & Medium low & 70 & $6 \%$ \\
AS16 & 9.0 & Super-macroporous & 42.5 & Ultralow & 80 & $1 \%$ \\
\hline
\end{tabular}



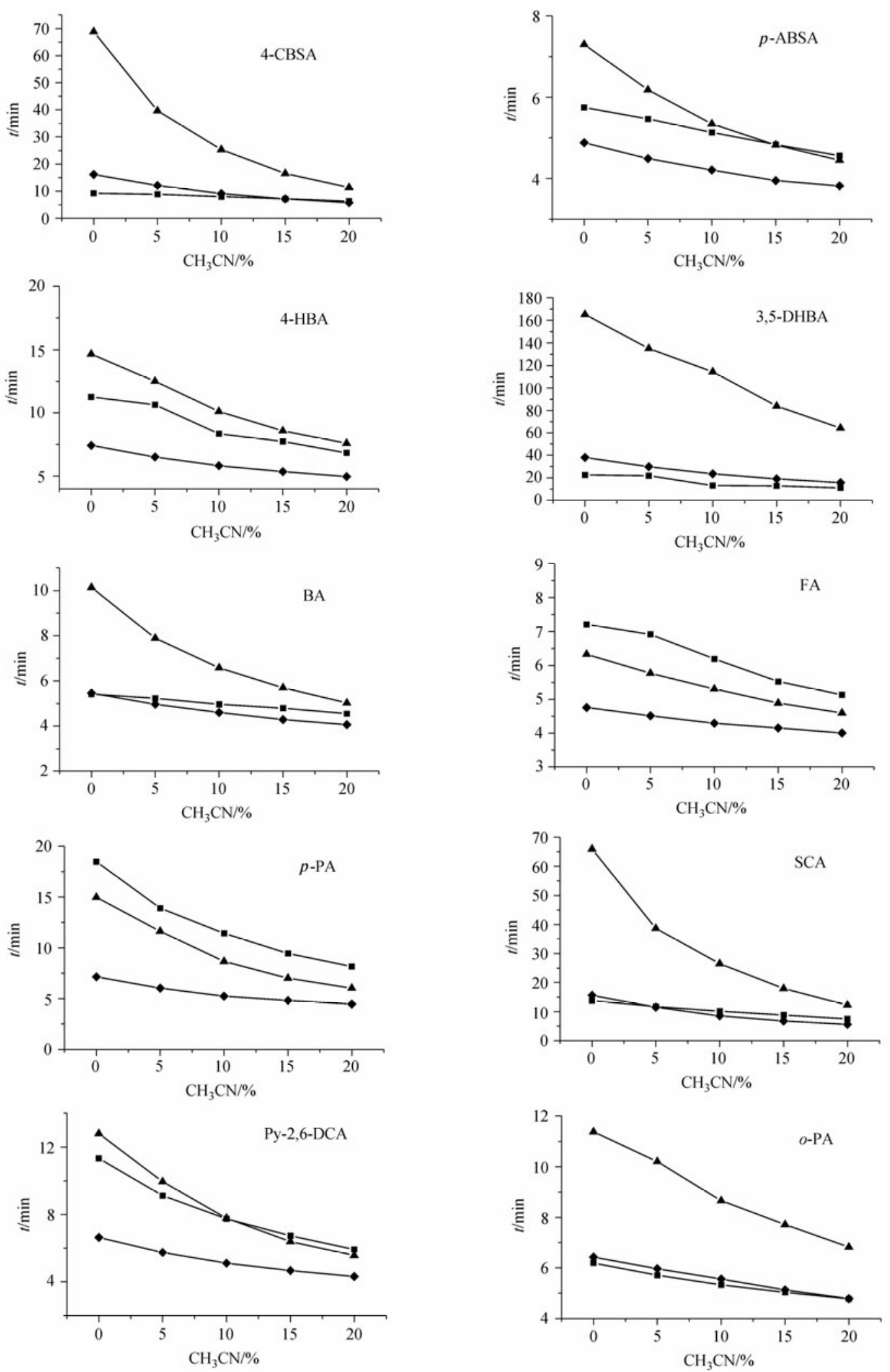

Figure 2 The effect of acetonitrile on the retention time at $30{ }^{\circ} \mathrm{C}$. $\Delta-\mathrm{AS} 11-\mathrm{HC} ;-\mathrm{AS} 16$; $-\mathrm{AS} 20$.

coating of AS20 column is aliphatic backbone. So the hydrophobicity function should be stronger in AS16 than in AS20. This point has also been proved in Figure 2 . It is obvious that rates of retention decrease for these six compounds are the smallest in AS20. Therefore, their retention is stronger in AS16 column than in AS20 column though the column capacity of AS20 is nearly twice as AS16.

The comparison study showed the $\pi-\pi$ bond action between the aromatic anions and stationary phase was weakened largely. This problem is evident for 4-CBSA, 3,5-DHBA and SCA. Their retention time was shortened 
largely because there was no $\pi$ electron in the AS20 column. This phenomenon is also seen in Figure 2. Their retention did not change with the content of acetonitrile increasing.

\section{The study of retention behavior in the IonPac AS20}

The above study has proved that the retentions of the selected compounds were weak in AS20. So we selected the IonPac AS20 as a column for their separation. The effects of $\mathrm{NaOH}$ concentration on their retention time were studied and the result is showed in Figure 3. It was showed that all analytes could be eluted from the column using lower concentration $\mathrm{NaOH}$. The gradient elution should be adopted to speed up the separation. So according to the Figure 3, the gradient eluent program for the separation of the ten organic acids was optimized. At first, the sodium hydroxide eluent was changed from 5 to $20 \mathrm{mmol} / \mathrm{L}$ within $20 \mathrm{~min}$ in a linear mode, then changed to $40 \mathrm{mmol} / \mathrm{L}$ within $10 \mathrm{~min}$ in a linear mode, and maintained at $40 \mathrm{mmol} / \mathrm{L}$ for $10 \mathrm{~min}$, and finally changed to $5 \mathrm{mmol} / \mathrm{L}$ at $40.1 \mathrm{~min}$ to equilibrate the system. The result showed that the ten compounds could be separated baselinely when the eluent was run in the gradient program. The chromatogram of the ten aromatic acids is shown in Figure 4. And their chromatographic parameters are listed in Table 2. The result showed that the peaks of the ten analytes were good in AS20 because the $\pi-\pi$ bond actions were weakened. The asymmetries

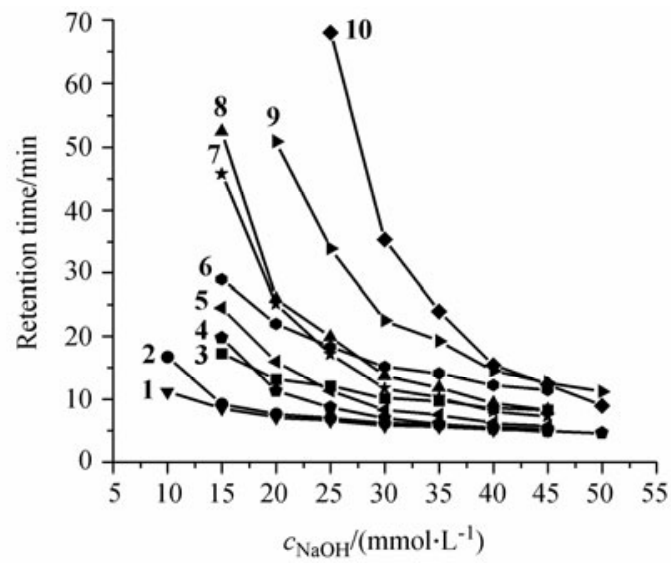

Figure 3 The effect of $\mathrm{NaOH}$ concentration on retention time in the AS20 column at $30{ }^{\circ} \mathrm{C}$. All ionic concentrations are $40 \mathrm{mg} / \mathrm{L}$ except for that of 4-CBSA, which is $12.5 \mathrm{mg} / \mathrm{L}$.

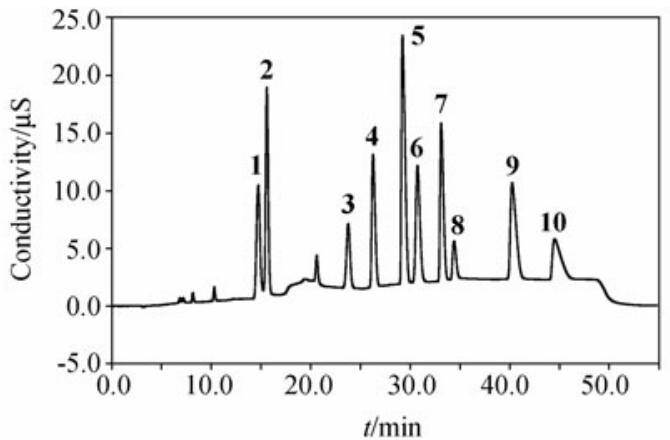

Figure 4 Chromatogram of the ten anions with the IonPac AS20 as a separating column and $\mathrm{NaOH}$ gradient eluent at $30{ }^{\circ} \mathrm{C}$. All ionic concentrations are $40 \mathrm{mg} / \mathrm{L}$ except for that of 4-CBSA, which is $12.5 \mathrm{mg} / \mathrm{L}$.

of the ten acids are in the range of 1.09-2.30. And the asymmetry of only one acid, 3,5-dihydroxybenzoic acid, is over 2.0. So the ever-present problem before, such as strong retention and the presence of tailed peak, would be solved when the AS20 column was used to separate aromatic acid. And the organic modifier was unnecessary.

\section{The effect of temperature on the retention behavior}

Temperature is one of factors affecting anion retention. So we studied the effect of temperature on their retention. As shown in Figure 5, which is chromatogram for the mixture of those ten compounds at various column temperatures from 30 to $45{ }^{\circ} \mathrm{C}$, the retention time

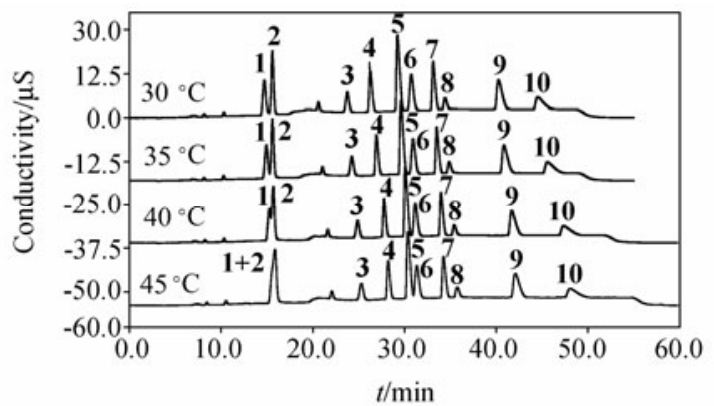

Figure 5 The effect of temperature on the retention behavior of the ten aromatic ionic compounds in the AS20 column. All ionic concentrations are $40 \mathrm{mg} / \mathrm{L}$ except for that of 4-CBSA, which is $12.5 \mathrm{mg} / \mathrm{L}$.

Table 2 Peak parameters of ten organic acids

\begin{tabular}{|c|c|c|c|c|c|c|}
\hline No. & Peak name & Retention time/min & Peak width/min & Resolution & Asymmetry & Plates \\
\hline 1 & $\mathrm{BA}$ & 14.72 & 0.64 & 1.52 & 1.20 & 8187 \\
\hline 2 & $p$-ABSA & 15.59 & 0.50 & 14.07 & 1.12 & 15607 \\
\hline 3 & 4-CBSA & 23.76 & 0.67 & 3.88 & 1.09 & 20388 \\
\hline 4 & $o$-PA & 26.26 & 0.62 & 4.47 & 1.44 & 28383 \\
\hline 5 & FA & 29.22 & 0.72 & 2.04 & 1.87 & 27525 \\
\hline 6 & SCA & 30.73 & 0.77 & 3.39 & 1.27 & 24953 \\
\hline 7 & Py-2,6-DCA & 33.11 & 0.64 & 2.00 & 1.66 & 43848 \\
\hline 8 & 4-HBA & 34.40 & 0.65 & 6.71 & 1.38 & 43184 \\
\hline 9 & $p$-PA & 40.24 & 1.10 & 3.19 & 1.77 & 22054 \\
\hline 10 & 3,5-DHBA & 44.52 & 1.64 & & 2.30 & 12332 \\
\hline
\end{tabular}


of almost all the ten compounds is increased with the temperature increase except for the $p$-ABSA. The retention of $p$-ABSA was independent of column temperature.

Van't Hoff plots are often used to show the relationship between retention value and temperature. van't Hoff equation is listed in the following:

$$
\ln k=-\Delta H^{\mathrm{o}} / R T+\Delta S^{\mathrm{o}} / R+\ln \phi
$$

where $T$ is the system temperature (Kelvin), $k$ is the capacity factor and it can be obtained mathematically by $\left(t_{\mathrm{R}}-t_{0}\right) / t_{0},\left(t_{\mathrm{R}}\right.$ and $t_{0}$ are the retention time of analyte and dead time respectively), $\Delta H^{\circ}$ and $\Delta S^{\mathrm{o}}$ are the enthalpy and entropy changes for the exchange reaction, $R$ and $\phi$ are both constants. They are the universal gas constant and column phase-ratio. vant' Hoff plots are those of $\ln k$ vs. 1/T, which are linear with the slope equal to $-\Delta H^{\circ} / R$ when enthalpy and entropy changes are temperature invariant. If the slope of linear plot is negative, the retention of the analyte is an endothermic process. If the slope is positive, the retention is an exothermic process. Linear regression parameters of the van't Hoff plots for all analytes are listed in Table 3. As shown in Table 3, the slopes are all negative, which indicates that the retentions of all the analytes are endothermic process. But the correlation coefficient of p-ABSA van't Hoff curve is 0.0537 , which indicates its retention is independent of column temperature.

Table 3 Linear regression data for plots of $\ln k$ vs. $1 / T\left(\times 10^{3}\right.$ $\mathrm{K}^{-1}$ ) of all the analytes with gradient eluent of $\mathrm{NaOH}$

\begin{tabular}{lccc}
\hline Analyte & Slope $=-\Delta H^{\circ} / R$ & $\begin{array}{c}\text { Intercept }= \\
\Delta S / R+\ln \phi\end{array}$ & $\begin{array}{c}\text { Correlation } \\
\text { coefficient }\left(r^{2}\right)\end{array}$ \\
\hline BA & -0.5031 & 2.9443 & 0.9786 \\
$p$-ABSA & -0.0119 & 1.3982 & 0.0537 \\
4-CBSA & -0.4508 & 3.354 & 0.971 \\
$o$-PA & -0.5178 & 3.69 & 0.98 \\
FA & -0.2723 & 2.999 & 0.9778 \\
SCA & -0.1346 & 2.6011 & 0.9365 \\
Py-2,6-DCA & -0.239 & 3.0283 & 0.9824 \\
4-HBA & -0.2666 & 3.1618 & 0.9807 \\
$p$-PA & -0.3269 & 3.5314 & 0.9787 \\
3,5-DHBA & -0.5707 & 4.4439 & 0.9811 \\
\hline
\end{tabular}

That an available column capacity became larger at increased temperature will mean prolonged retention time if ions of interest penetrate into the pores more easily at elevated temperatures. So the AS20 column capacity at different temperature was studied. The processes are as follows: firstly, pump the $10 \mathrm{mmol} / \mathrm{L} \mathrm{HCl}$ into the AS20+AG20 column for $45 \mathrm{~min}$ to saturate the column adequately. And secondly, wash the column with water until about $\mathrm{pH}$ 7. Thirdly, elute chloride using $60 \mathrm{mmol} / \mathrm{L} \mathrm{NaOH}$ and collect the effluent into the volumetric flask. Finally, dilute the effluent into $25 \mathrm{~mL}$ with high purity water and determine the chloride. The concentration of the chloride, which can reflect the capacity of column indirectly, is respectively $86.8,86.5$, 85.3 and $85.0 \mathrm{mg} / \mathrm{L}$ at $25,30,35$ and $40{ }^{\circ} \mathrm{C}$. The result showed that the column capacity was independent of column temperature. So the reason for increasing retention time with increasing temperature is not the available column capacity increase but the presence of endothermic process.

\section{Conclusion}

In this paper, the retention behavior of ten aromatic ionic compounds on three anion-exchange columns was compared. The result showed that IonPac AS20 could be used to separate aromatic ionic compounds and it was more appreciated than the other two columns. The retention of the aromatic compounds can be shortened because the hydrophobic interaction is weakened.

\section{References and notes}

1 Waksmundzka-Hajnos, M. J. Chromatogr., B 1998, 717, 93.

2 http://www.scorecard.org/chemical-profiels/index.tcl

3 Grechishkina, O. J. Chromatogr., A 2002, 948, 65.

4 Alonso, M. C.; Barceló, D. Anal. Chim. Acta 1999, 400, 211.

5 Storm, T.; Reemtsma, T.; Jekel, M. J. Chromatogr., A 1999, 854,175 .

6 Loos, R.; Alonso, M. C.; Barcelo, D. J. Chromatogr., A 2000, 890, 225.

7 Alonso, M. C.; Tirapu, L.; Ginebreda, A.; Barceló, D. Environ. Pollut. 2005, 137, 253.

8 Chen, S. F.; Mowery, R. A.; Castleberry, V. A.; Walsum, G. P.; Chambliss, C. K. J. Chromatogr., A 2006, 1104, 54.

9 Sarkar, A. B.; Kochak, G. M. Carbohydr. Polym. 2005, 59, 305.

10 Adams, M. A.; Chen, Z.; Landman, P.; Colmer, T. D. Anal. Biochem. 1999, 264, 77.

11 Chen, Z. L.; Adams, M. A. Anal. Chim. Acta 1999, 386, 249.

12 Chen, Z. L.; Adams, M. A. J. Liq. Chromatogr. Relat. Technol. 1998, 21, 2435.

13 Glod, B. K. Neurochem. Res. 1997, 22, 1237.

14 Alcázar, A.; Fernández-Cáceres, P. L.; Martín, M. J.; Pablos, F.; González, A. G. Talanta 2003, 61, 95.

15 Masson, P. J. Chromatogr., A 2000, 881, 387.

16 Lu, Z.; Liu, Y.; Barreto, V.; Pohl, C.; Avdalovic, N.; Joyce, R.; Newton, B. J. Chromatogr., A 2002, 956, 129.

17 Sarzanini, C.; Bruzzoniti, M. C.; Hajós, P. J. Chromatogr., A 2000, 867, 131.

18 Chen, J. J. Chromatogr., A 1996, 739, 273.

19 Nait, L. M.; Kildew, B. R.; Saari-Nordhaus, R. J. Chromatogr., A 1996, 739, 99.

20 Fritz, J. S. J. Chromatogr., A 2005, 1085, 8.

21 Wagner, H. P.; Pepich, B. V.; Pohl, C.; Later, D.; Joyce, R.; Srinivasan, K.; Thomas, D.; Woodruff, A.; DeBorba, B.; Munch, D. J. J. Chromatogr., A 2006, 1118, 85. 